\title{
Front Matter: Volume 8640
}

, "Front Matter: Volume 8640," Proc. SPIE 8640, Novel In-Plane Semiconductor Lasers XII, 864001 (8 April 2013); doi: 10.1117/12.2021932

SPIE. Event: SPIE OPTO, 2013, San Francisco, California, United States 


\title{
PROCEEDINGS OF SPIE
}

\section{Novel In-Plane \\ Semiconductor Lasers XII}

\author{
Alexey A. Belyanin \\ Peter M. Smowton \\ Editors
}

4-7 February 2013

San Francisco, California, United States

Sponsored and Published by

SPIE 
The papers included in this volume were part of the technical conference cited on the cover and title page. Papers were selected and subject to review by the editors and conference program committee. Some conference presentations may not be available for publication. The papers published in these proceedings reflect the work and thoughts of the authors and are published herein as submitted. The publisher is not responsible for the validity of the information or for any outcomes resulting from reliance thereon.

Please use the following format to cite material from this book:

Author(s), "Title of Paper," in Novel In-Plane Semiconductor Lasers XII, edited by

Alexey A. Belyanin, Peter M. Smowton, Proceedings of SPIE Vol. 8640 (SPIE, Bellingham, WA, 2013) Article CID Number.

ISSN: 0277-786X

ISBN: 9780819494092

Published by

SPIE

P.O. Box 10, Bellingham, Washington 98227-0010 USA

Telephone +1 3606763290 (Pacific Time) · Fax +1 3606471445

SPIE.org

Copyright (@ 2013, Society of Photo-Optical Instrumentation Engineers.

Copying of material in this book for internal or personal use, or for the internal or personal use of specific clients, beyond the fair use provisions granted by the U.S. Copyright Law is authorized by SPIE subject to payment of copying fees. The Transactional Reporting Service base fee for this volume is $\$ 18.00$ per article (or portion thereof), which should be paid directly to the Copyright Clearance Center (CCC), 222 Rosewood Drive, Danvers, MA 01923. Payment may also be made electronically through CCC Online at copyright.com. Other copying for republication, resale, advertising or promotion, or any form of systematic or multiple reproduction of any material in this book is prohibited except with permission in writing from the publisher. The CCC fee code is 0277-786X/13/\$18.00.

Printed in the United States of America.

Publication of record for individual papers is online in the SPIE Digital Library.

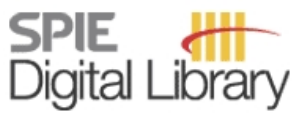

SPIEDigitalLibrary.org

Paper Numbering: Proceedings of SPIE follow an e-First publication model, with papers published first online and then in print and on CD-ROM. Papers are published as they are submitted and meet publication criteria. A unique, consistent, permanent citation identifier (CID) number is assigned to each article at the time of the first publication. Utilization of CIDs allows articles to be fully citable as soon as they are published online, and connects the same identifier to all online, print, and electronic versions of the publication. SPIE uses a six-digit CID article numbering system in which:

- The first four digits correspond to the SPIE volume number.

- The last two digits indicate publication order within the volume using a Base 36 numbering

system employing both numerals and letters. These two-number sets start with 00, 01, 02, 03, 04, $05,06,07,08,09,0 A, 0 B \ldots$. 0Z, followed by 10-1Z, 20-2Z, etc.

The CID Number appears on each page of the manuscript. The complete citation is used on the first page, and an abbreviated version on subsequent pages. Numbers in the index correspond to the last two digits of the six-digit CID Number. 


\title{
Contents
}

\author{
ix Conference Committee \\ xi Group IV photonics for the mid infrared (Plenary Paper) [8629-1] \\ R. Soref, The Univ. of Massachusetts at Boston (United States) \\ xxvii Light in a twist: optical angular momentum (Plenary Paper) [8637-2] \\ M. J. Padgett, Univ. of Glasgow (United Kingdom)
}

\section{COMMS: MATERIALS TO INTEGRATION}

864004 High modal gain $1.5 \mu \mathrm{m}$ InP based quantum dot lasers: dependence of static properties on the active layer design [8640-2]

V. Sichkovskyi, V. Ivanov, J. P. Reithmaier, Univ. Kassel (Germany)

864005 Effect of optical waveguiding mechanism on the lasing action of chirped InAs/AIGalnAs/InP quantum dash lasers [8640-4]

M. Z. M. Khan, T. K. Ng, King Abdullah Univ. of Science and Technology (Saudi Arabia);

C.-S. Lee, P. Bhattacharya, Univ. of Michigan (United States); B. S. Ooi, King Abdullah Univ.

of Science and Technology (Saudi Arabia)

\section{DFBS AND DBRS}

864008 Monolithic wide tunable laser diodes for gas sensing at $2100 \mathrm{~nm}$ [8640-7]

N. Koslowski, A. Heger, K. Roessner, M. Legge, L. Hildebrandt, J. Koeth, nanoplus

Nanosystems and Technologies GmbH (Germany)

864009 Narrow-linewidth three-electrode regrowth-free semiconductor DFB lasers with uniform surface grating [8640-8]

K. Dridi, A. Benhsaien, A. Akrout, Univ. of Ottawa (Canada); J. Zhang, CMC Microsystems (Canada); T. Hall, Univ. of Ottawa (Canada)

8640 OA Sub-MHz linewidth of $633 \mathrm{~nm}$ diode lasers with internal surface DBR gratings [8640-9] D. Feise, G. Blume, J. Pohl, B. Sumpf, Ferdinand-Braun-Institut (Germany); H. Thiem, M. Reggentin, J. Wiedmann, Eagleyard Photonics GmbH (Germany); K. Paschke, Ferdinand-Braun-Institut (Germany)

RED, GREEN, BLUE

$8640 \mathrm{OD} \quad 654 \mathrm{~nm}$ broad area lasers for QCW operation with a maximal facet load of $76 \mathrm{~mW} / \mu \mathrm{m}$ [8640-12]

B. Sumpf, M. Pohl, W. Pittroff, R. Staske, G. Erbert, G. Tränkle, Ferdinand-Braun-Institut (Germany) 
8640 OE Highly reliable operation of $638-\mathrm{nm}$ broad stripe laser diode with high wall-plug efficiency for display applications [8640-13]

T. Yagi, N. Shimada, T. Nishida, H. Mitsuyama, M. Miyashita, Mitsubishi Electric Corp. (Japan)

8640 OF Continuous-wave operation of green/yellow laser diodes based on BeZnCdSe quantum wells (Invited Paper) [8640-14]

R. Akimoto, T. Hasama, H. Ishikawa, National Institute of Advanced Industrial Science and Technology (Japan); J. Kasai, S. Fujisaki, S. Tanaka, S. Tsuji, Hitachi Ltd. (Japan)

8640 OG Power blue and green laser diodes and their applications (Invited Paper) [8640-15]

T. Hager, U. Strauß, C. Eichler, C. Vierheilig, S. Tautz, G. Brüderl, B. Stojetz, T. Wurm,

A. Avramescu, A. Somers, J. Ristic, S. Gerhard, A. Lell, S. Morgott, OSRAM Opto

Semiconductors GmbH (Germany); O. Mehl, OSRAM GmbH (Germany)

NITRIDES

$8640 \mathrm{OH}$ Passive mode-locking in the cavity of monolithic GaN-based multi-section laser diodes [8640-16]

T. Weig, Fraunhofer Institute for Applied Solid State Physics (Germany); U. T. Schwarz,

Fraunhofer Institute for Applied Solid State Physics (Germany) and Univ. of Freiburg (Germany); L. Sulmoni, J.-M. Lamy, J.-F. Carlin, N. Grandjean, Ecole Polytechnique Fédérale de Lausanne (Switzerland); D. Boiko, Ctr. Suisse d'Electronique et de Microtechnique (Switzerland)

8640 0J InGaN/GaN quantum dot blue and green lasers (Invited Paper) [8640-18]

P. Bhattacharya, A. Banerjee, T. Frost, Univ. of Michigan (United States)

MID-IR LASERS AND OPTICS

$86400 Q$ Recent progress in development of InAs-based interband cascade lasers (Invited Paper) [8640-25]

R. Q. Yang, L. Li, L. Zhao, Y. Jiang, Z. Tian, H. Ye, R. T. Hinkey, C. Niu, T. D. Mishima,

M. B. Santos, J. C. Keay, M. B. Johnson, The Univ. of Oklahoma (United States); K. Mansour, Jet Propulsion Lab. (United States)

8640 OR Extremely temperature insensitive continuous-wave broadband quantum cascade lasers [8640-26]

K. Fujita, M. Yamanishi, S. Furuta, T. Dougakiuchi, A. Sugiyama, T. Edamura, Hamamatsu Photonics K.K. (Japan)

\section{SILICON BASED LASERS}

8640 OU Optimization of the hybrid silicon photonic integrated circuit platform (Invited Paper) [8640-29]

M. J. R. Heck, M. L. Davenport, S. Srinivasan, J. Hulme, J. E. Bowers, Univ. of California, Santa Barbara (United States)

iv 
MICROCAVITY AND PHOTONIC CRYSTAL

8640 OY Electrical injection schemes for nanolasers [8640-34]

A. Lupi, I.-S. Chung, K. Yvind, Technical Univ. of Denmark (Denmark)

$86400 Z$ Towards a photonic crystal mode-locked laser [8640-35]

K. Leedle, A. Janjua, S. Paik, M. J. Schnitzer, J. S. Harris, Stanford Univ. (United States)

\section{THZ QCLS}

864014 THz quantum cascade lasers for operation above cryogenic temperatures (Invited Paper) [8640-40]

M. A. Belkin, K. Vijayraghavan, The Univ. of Texas at Austin (United States); A. Vizbaras, Technische Univ. München (Germany); and Brolis Semiconductors Ltd. (Lithuania); A. Jiang, The Univ. of Texas at Austin (United States); F. Demmerle, G. Boehm, R. Meyer, M.-C. Amann, A. Matyas, R. Chashmahcharagh, P. Lugli, C. Jirauschek, Technische Univ. München (Germany); Z. R. Wasilewski, Univ. of Waterloo (Canada)

\section{LONG-WAVELENGTH MID-IR AND THZ QCLS}

864015 Long-wavelength $(\lambda \approx 12-16 \mu \mathrm{m})$ and cascaded transition quantum cascade lasers (Invited Paper) [8640-41]

X. Huang, Y. Chiu, J. L. Zhang, Princeton Univ. (United States); W. O. Charles, Princeton Univ. (United States) and Phononic Devices, Inc. (United States); V. E. Tokranov, S. Oktyabrsky, Univ. at Albany, SUNY (United States); C. F. Gmachl, Princeton Univ. (United States)

864016 Room temperature continuous wave operation of long wavelength (9-11 $\mu \mathrm{m})$ distributed feedback quantum cascade lasers for glucose detection [8640-42]

F. Xie, C. Caneau, H. P. LeBlanc, S. Coleman, M.-T. Ho, L. C. Hughes, C. Zah, Corning Inc. (United States)

864018 Towards nanowire-based terahertz quantum cascade lasers: prospects and technological challenges [8640-44]

M. Krall, M. Brandstetter, C. Deutsch, H. Detz, T. Zederbauer, A. M. Andrews, W. Schrenk, G. Strasser, K. Unterrainer, Technische Univ. Wien (Austria)

\section{HIGH-POWER AND TUNABLE QCLS}

864019 Multiwatt long wavelength quantum cascade lasers based on high strain composition with $70 \%$ injection efficiency [8640-45]

A. Lyakh, R. Maulini, A. G. Tsekoun, R. Go, C. K. N. Patel, Pranalytica, Inc. (United States)

8640 IE Single-mode quantum cascade lasers with asymmetric Mach-Zehnder interferometer type Fabry-Perot cavity [8640-50]

P. Q. Liu, Princeton Univ. (United States); X. Wang, AdTech Optics, Inc. (United States);

C. F. Gmachl, Princeton Univ. (United States) 
$8640 \mathrm{IF}$ Analysis of bulk and facet failures in AIGaAs-based high-power diode lasers (Invited Paper) [8640-51]

J. W. Tomm, M. Hempel, Max-Born-Institut für Nichtlineare Optik und Kurzzeitspektroskopie (Germany); F. La Mattina, EMPA (Switzerland); F. M. Kießling, Leibniz-Institut für Kristallzüchtung (Germany); T. Elsaesser, Max-Born-Institut für Nichtlineare Optik und Kurzzeitspektroskopie (Germany)

8640 IG Catastrophic degradation in high-power InGaAs-AIGaAs strained quantum well lasers and InAs-GaAs quantum dot lasers [8640-52]

Y. Sin, S. LaLumondiere, B. Foran, N. Ives, N. Presser, W. Lotshaw, S. C. Moss, The Aerospace Corp. (United States)

$8640 \mathrm{1H}$ Comparison of catastrophic optical damage in InP/(AI)GalnP quantum dot and quantum well diode lasers [8640-53]

S. N. Elliott, Cardiff Univ. (United Kingdom); M. Hempel, Max-Born-Institut für Nichtlineare Optik und Kurzzeitspektroskopie (Germany); S. Shutts, Cardiff Univ. (United Kingdom);

U. Zeimer, Ferdinand-Braun-Institut (Germany); P. M. Smowton, Cardiff Univ. (United Kingdom); J. W. Tomm, Max-Born-Institut für Nichtlineare Optik und Kurzzeitspektroskopie (Germany)

864011 Multi-spectral investigation of bulk and facet failures in high-power single emitters at 980 nm (Invited Paper) [8640-54]

D. Yanson, M. Levy, M. Shamay, S. Cohen, L. Shkedy, Y. Berk, R. Tessler, G. Klumel, N. Rappaport, Y. Karni, SCD Semiconductor Devices (Israel)

$86401 \mathrm{~J} 1120 \mathrm{~nm}$ highly brilliant laser sources for SHG-modules in bio-analytics and spectroscopy [8640-55]

K. Paschke, C. Fiebig, G. Blume, F. Bugge, J. Fricke, G. Erbert, Ferdinand-Braun-Institut (Germany)

\section{HIGH POWER II}

$86401 \mathrm{~L} \quad \mathbf{7 6 \%}$ efficient cryogenically-cooled eyesafe diode laser for resonant pumping of Er-doped gain media [8640-57]

Z. Chen, W. Dong, X. Guan, S. Elim, S. Zhang, M. Grimshaw, M. Devito, P. Leisher, M. Kanskar, nLIGHT Corp. (United States)

8640 1M Dynamic response of a monolithic master-oscillator power-amplifier at $1.5 \mu \mathrm{m}$ [8640-59] P. Adamiec, B. Bonilla, A. Consoli, J. M. G. Tijero, S. Aguilera, I. Esquivias, M. Vilera, Univ. Politécnica de Madrid (Spain); J. Javaloyes, Univ. de les Illes Balears (Spain); S. Balle, Institut Mediterrani d'Estudis Avançats (Spain)

8640 iN Dynamics of high power gain switched DFB RW laser under high current pulse excitation on a nanosecond time scale [8640-60]

A. Klehr, S. Schwertfeger, H. Wenzel, T. Hoffmann, A. Liero, R. Staske, O. Brox, G. Erbert,

G. Tränkle, Ferdinand-Braun-Institut (Germany) 


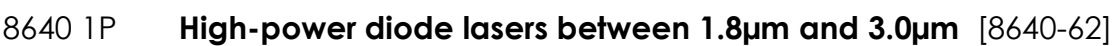

S. Hilzensauer, J. Gilly, P. Friedmann, m2k-laser GmbH (Germany); M. Werner, M. Traub, Fraunhofer Institute for Laser Technology (Germany); S. Patterson, DILAS Diodenlaser Inc. (United States); J. Neukum, DILAS Diodenlaser GmbH (Germany); M. T. Kelemen, m2k-laser GmbH (Germany)

8640 is Analysis of thermally activated leakage current in a low-threshold-current quantumcascade laser emitting at $3.9 \mu \mathrm{m}$ [8640-65]

Y. V. Flores, G. Monastyrskyi, M. Elagin, M. P. Semtsiv, W. T. Masselink, Humboldt-Univ. zu Berlin (Germany)

POSTER SESSION

8640 iU 980-nm external-cavity passively mode-locked laser with extremely narrow RF linewidth [8640-67]

Y. Ding, Univ. of Dundee (United Kingdom) and State Key Lab. on Integrated Optoelectronics (China); W. Ji, J. Chen, S. Zhang, X. Wang, Beijing Univ. of Technology (China); H. Wang, H. Ni, J. Pan, Institute of Semiconductors (China); B. Cui, Beijing Univ. of Technology (China); M. A. Cataluna, Univ. of Dundee (United Kingdom)

$8640 \mathrm{lV}$ Transverse mode control in quantum cascade lasers via lossy lateral constrictions [8640-68] P. M. Bouzi, P. Q. Liu, N. Aung, Princeton Univ. (United States); X. Wang, J.-Y. Fan, M. Troccoli, AdTech Optics, Inc. (United States); C. F. Gmachl, Princeton Univ. (United States)

Author Index 
Proc. of SPIE Vol. $8640864001-8$

Downloaded From: https://www.spiedigitallibrary.org/conference-proceedings-of-spie on 26 Apr 2023 Terms of Use: https://www.spiedigitallibrary.org/terms-of-use 


\section{Conference Committee}

Symposium Chair

David L. Andrews, University of East Anglia Norwich (United Kingdom)

Symposium Cochairs

Alexei L. Glebov, OptiGrate Corporation (United States)

Klaus P. Streubel, OSRAM GmbH (Germany)

Program Track Chair

Klaus P. Streubel, OSRAM GmbH (Germany)

Conference Chairs

Alexey A. Belyanin, Texas A\&M University (United States)

Peter M. Smowton, Cardiff University (United Kingdom)

Conference Program Committee

Yasuhiko Arakawa, The University of Tokyo (Japan)

Dan Botez, University of Wisconsin-Madison (United States)

Federico Capasso, Harvard School of Engineering and Applied Sciences (United States)

Gary A. Evans, Southern Methodist University (United States)

Claire F. Gmachl, Princeton University (United States)

Michael Kneissl, Technische Universität Berlin (Germany)

Hui Chun Liu, Shanghai Jiao Tong University (China)

Luke J. Mawst, University of Wisconsin-Madison (United States)

Jerry R. Meyer, U.S. Naval Research Laboratory (United States)

Richard V. Penty, University of Cambridge (United Kingdom)

Johann Peter Reithmaier, Universität Kassel (Germany)

Haisheng Rong, Intel Corporation (United States)

Nelson Tansu, Lehigh University (United States)

Shinji Tsuji, Hitachi, Ltd. (Japan)

Kresten Yvind, Technical University of Denmark (Denmark)

Session Chairs

1 Comms: Materials to Integration

Nelson Tansu, Lehigh University (United States) 
2 DFBs and DBRs

Johann Peter Reithmaier, Universität Kassel (Germany)

3 Red, Green, Blue

Peter M. Smowton, Cardiff University (United Kingdom)

$4 \quad$ Nitrides

Michael Kneissl, Technische Universität Berlin (Germany)

5 Mid-IR QCLS

Alexey A. Belyanin, Texas A\&M University (United States)

6 Mid-IR Lasers and Optics

Jerry R. Meyer, U.S. Naval Research Laboratory (United States)

7 Silicon Based Lasers

Haisheng Rong, Intel Corporation (United States)

8 Microcavity and Photonic Crystal

Weng W. Chow, Sandia National Laboratories (United States)

$9 \mathrm{THz} Q \mathrm{CLS}$

Jérôme Faist, ETH Zürich (Switzerland)

10 Long-Wavelength Mid-IR and THz QCLs

Mikhail A. Belkin, The University of Texas at Austin (United States)

11 High-Power and Tunable QCLs

Pietro Malara, Istituto Nazionale di Ottica (Italy)

12 High Power I

Gary A. Evans, Southern Methodist University (United States)

13 High Power II

Paul Crump, Ferdinand-Braun-Institut (Germany)

14 Short-Wavelength Mid-IR QCLs and Diode Lasers

Feng Xie, Corning Incorporated (United States) 